\title{
TABLE OF THE ZEROS OF THE LEGENDRE POLYNOMIALS \\ OF ORDER 1-16 AND THE WEIGHT COEFFICIENTS FOR GAUSS' MECHANICAL QUADRATURE FORMULA ${ }^{1}$
}

\author{
ARNOLD N. LOWAN, NORMAN DAVIDS AND ARTHUR LEVENSON
}

Gauss' method of mechanical quadrature has the advantage over most methods of numerical integration in that it requires about half the number of ordinate computations. This is desirable when such computations are very laborious, or when the observations necessary to determine the average value of a continuously varying physical quantity are very costly. Gauss' classical result ${ }^{2}$ states that, for the range $(-1,+1)$, the "best" accuracy with $n$ ordinates is obtained by choosing the corresponding abscissae at the zeros $x_{1}, \cdots, x_{n}$ of the Legendre polynomials $P_{n}(x)$. With each $x_{i}$ is associated a constant $a_{i}$ such that

$$
\int_{-1}^{1} f(x) d x \sim a_{1} f\left(x_{1}\right)+a_{2} f\left(x_{2}\right)+\cdots+a_{n} f\left(x_{n}\right) .
$$

The accompanying table computed by the Mathematical Tables Project gives the roots $x_{i}$ for each $P_{n}(x)$ up to $n=16$, and the corresponding weight coefficients $a_{i}$, to 15 decimal places.

The first such table, computed by Gauss gave 16 places up to $n=7 .^{3}$ More recently work was done by Nyström, ${ }^{4}$ who gave 7 decimals up to $n=10$, but for the interval $(-1 / 2,+1 / 2)$. B. de F. Bayly has given the roots and coefficients of $P_{12}(x)$ to 13 places. ${ }^{5}$

The Gaussian quadrature formula for evaluating an integral with arbitrary limits $(p, q)$ is given by

Presented to the Society, October 25, 1941, under the title Tables for Gauss' mechanical quadrature formula; received by the editors December 18, 1941 .

${ }_{1}$ The results reported here were obtained in the course of the work done by the Mathematical Tables Project conducted by the Work Projects Administration for New York City under the sponsorship of the National Bureau of Standards, Dr. Lyman J. Briggs, Director.

2 Methodus nova integralium valores per approximationen inveniendi, Commentationes Societatis Regiae Scientiarum Gottingensis Recentiores, vol. 3 (1814), or Werke, vol. 3, pp. 193-195.

${ }^{3}$ It may be found reproduced in Heine's Kugelfunctionen, vol. 2, 1881, p. 15, or Hobson, Spherical Harmonics, pp. 80-81.

${ }^{4}$ Nyström, Acta Mathematica, vol. 54 (1930), p. 191.

5 B. de F. Bayly, Biometrika, vol. 30 (1938), pp. 193-194. 


$$
\int_{p}^{q} f(x) d x=\frac{q-p}{2} \sum_{i=1}^{n} a_{i} f\left(x_{i} \frac{q-p}{2}+\frac{q+p}{2}\right)+R_{n}(f)
$$

where $x_{i}=x_{i, n}$ is the $i$ th root of $P_{n}(x)$ and

$$
a_{i}=a_{i, n}=\frac{1}{P_{n}^{\prime}\left(x_{i}\right)} \int_{-1}^{1} \frac{P_{n}(x)}{x-x_{i}} d x
$$

We have

$$
x_{i}=-x_{n-i+1}, a_{i}=a_{n-i+1},
$$

so that only half the values need be tabulated.

It is well known that if $f_{2 n-1}(x)$ is an arbitrary polynomial of degree at most $2 n-1$, then

$$
R_{n}\left(f_{2 n-1}\right)=0,
$$

that is, formula (1) is exact. Thus Gauss' formula with $n$ ordinates provides an approximation as good as would have been obtained by using a polynomial of degree $2 n-1$.

If $f(x)$ has a continuous derivative of order $2 n$ in the interval $(p, q)$, then $^{6}$

$$
R_{n}=\frac{f^{(2 n)}(\xi)}{(2 n) ! k_{n}^{2}}
$$

where $\xi$ is a point in the interval $(p, q)$ and $k_{n}$ is the normalizing factor for $P_{n}(x)$, which is equal to

$$
\left(\frac{2 n+1}{q-p}\right)^{1 / 2}
$$

The roots were calculated by successive approximations, combining synthetic division with Newton's tangent formula. They were checked by using the relations between roots and coefficients.

By suitable transformations of formula (3), the computation of the weight coefficients $a_{i}$ was made to depend on that of the roots, in particular on the values of the successive quotients obtained in the process of synthetic division. These weight coefficients were checked by putting $f(x) \equiv 1$ in formula (1), giving

$$
2=a_{1}+a_{2}+\cdots+a_{n} .
$$

The sum of the $a_{i}$ 's was required not to differ from 2 by more than one unit in the 17 th place.

6 This expression is due to Markoff. See Szegö, Orthogonal Polynomials, American Mathematical Society Colloquium Publications, vol. 23, 1939, p. 369. 
TABLE$$
x_{1}=0.577350269189626
$$$$
x_{0}=0.000000000000000
$$$$
x_{1}=0.774596669241483
$$

$x_{1}=0.339981043584856$ $x_{2}=0.861136311594053$

$x_{0}=0.000000000000000$ $x_{1}=0.538469310105683$ $x_{2}=0.906179845938664$

$x_{1}=0.238619186083197$ $x_{2}=0.661209386466265$ $x_{3}=0.932469514203152$

$x_{0}=0.000000000000000$ $x_{1}=0.405845151377397$ $x_{2}=0.741531185599394$ $x_{3}=0.949107912342759$

$x_{1}=0.183434642495650$ $x_{2}=0.525532409916329$ $x_{3}=0.796666477413627$ $x_{4}=0.960289856497536$

$x_{0}=0.000000000000000$ $x_{1}=0.324253423403809$ $x_{2}=0.613371432700590$ $x_{3}=0.836031107326636$ $x_{4}=0.968160239507626$

$x_{1}=0.148874338981631$ $x_{2}=0.433395394129247$ $x_{3}=0.679409568299024$ $x_{4}=0.865063366688985$ $x_{5}=0.973906528517172$

$x_{0}=0.000000000000000$ $x_{1}=0.269543155952345$ $x_{2}=0.519096129110681$ $x_{3}=0.730152005574049$ $x_{4}=0.887062599768095$ $x_{5}=0.978228658146057$

$$
\begin{aligned}
& n=2 \\
& n=3 \\
& a_{1}=1.000000000000000 \\
& a_{0}=0.888888888888889 \\
& a_{1}=0.555555555555556 \\
& a_{1}=0.652145154862546 \\
& a_{2}=0.347854845137454 \\
& n=5 \\
& n=6
\end{aligned}
$$

$$
n=7
$$

$a_{0}=0.417959183673469$

$a_{1}=0.381830050505119$

$a_{2}=0.279705391489277$

$a_{3}=0.129484966168870$

$$
n=8
$$

$a_{1}=0.362683783378362$

$a_{2}=0.313706645877887$

$a_{3}=0.222381034453374$

$a_{4}=0.101228536290376$

$$
n=9
$$

$a_{0}=0.330239355001260$

$a_{1}=0.312347077040003$

$a_{2}=0.260610696402935$

$a_{3}=0.180648160694857$

$a_{4}=0.081274388361574$

$n=10$

$a_{1}=0.295524224714753$

$a_{2}=0.269266719309996$

$a_{3}=0.219086362515982$

$a_{4}=0.149451349150581$

$a_{5}=0.066671344308688$

$n=11$

$a_{0}=0.272925086777901$

$a_{1}=0.262804544510247$

$a_{2}=0.233193764591990$

$a_{3}=0.186290210927734$

$a_{4}=0.125580369464905$

$a_{5}=0.055668567116174$ 


\section{TABLE-Continued}

$x_{1}=0.125333408511469$

$x_{2}=0.367831498918180$

$x_{3}=0.587317954286617$

$x_{4}=0.769902674194305$

$x_{5}=0.904117256370475$

$x_{6}=0.981560634246719$

$x_{0}=0.000000000000000$

$x_{1}=0.230458315955135$

$x_{2}=0.448492751036447$

$x_{3}=0.642349339440340$

$x_{4}=0.801578090733310$

$x_{5}=0.917598399222978$

$x_{6}=0.984183054718588$

$x_{1}=0.108054948707344$

$x_{2}=0.319112368927890$

$x_{3}=0.515248636358154$

$x_{4}=0.687292904811685$

$x_{5}=0.827201315069765$

$x_{6}=0.928434883663574$

$x_{7}=0.986283808696812$

$x_{0}=0.000000000000000$

$x_{1}=0.201194093997435$

$x_{2}=0.394151347077563$

$x_{3}=0.570972172608539$

$x_{4}=0.724417731360170$

$x_{5}=0.848206583410427$

$x_{6}=0.937273392400706$

$x_{7}=0.987992518020485$

$x_{1}=0.095012509837637$

$x_{2}=0.281603550779259$

$x_{3}=0.458016777657227$

$x_{4}=0.617876244402644$

$x_{5}=0.755404408355003$

$x_{6}=0.865631202387832$

$x_{7}=0.944575023073233$

$x_{8}=0.989400934991650$

$$
n=12
$$

$a_{1}=0.249147045813403$

$a_{2}=0.233492536538355$

$a_{3}=0.203167426723066$

$a_{4}=0.160078328543346$

$a_{5}=0.106939325995318$

$a_{6}=0.047175336386512$

$n=13$

$a_{0}=0.232551553230874$

$a_{1}=0.226283180262897$

$a_{2}=0.207816047536889$

$a_{3}=0.178145980761946$

$a_{4}=0.138873510219787$

$a_{5}=0.092121499837728$

$a_{6}=0.040484004765316$

$n=14$

$a_{1}=0.215263853463158$

$a_{2}=0.205198463721296$

$a_{3}=0.185538397477938$

$a_{4}=0.157203167158194$

$a_{5}=0.121518570687903$

$a_{6}=0.080158087159760$

$a_{7}=0.035119460331752$

$n=15$

$a_{0}=0.202578241925561$

$a_{1}=0.198431485327111$

$a_{2}=0.186161000015562$

$a_{3}=0.166269205816994$

$a_{4}=0.139570677926154$

$a_{5}=0.107159220467172$

$a_{6}=0.070366047488108$

$a_{7}=0.030753241996117$

$n=16$

$a_{1}=0.189450610455069$

$a_{2}=0.182603415044924$

$a_{3}=0.169156519395003$

$a_{4}=0.149595988816577$

$a_{5}=0.124628971255534$

$a_{6}=0.095158511682493$

$a_{7}=0.062253523938648$

$a_{8}=0.027152459411754$

Example. Let it be desired to find $I=\int_{1}^{2} d x / x$. By formula (2)

$$
I \sim \sum \frac{a_{k}}{x_{k}^{3}}
$$


Taking $n=10$, we have

$$
\begin{aligned}
I \sim & \frac{0.295524224714753}{2.851125661018369}+\frac{0.269266719309996}{2.566604605870753} \\
& +\frac{0.219086362515982}{2.320590431700976}+\frac{0.149451349150581}{2.134936633311015} \\
& +\frac{0.066671344308688}{2.026093471482828}+\frac{0.295524224714753}{3.148874338981631} \\
& +\frac{0.269266719309996}{3.433395394129247}+\frac{0.219086362515982}{3.679409568299024} \\
& +\frac{0.149451349150581}{3.865063366688985}+\frac{0.066671344308688}{3.973906528517172} \\
= & 0.693147180559947 .
\end{aligned}
$$

This agrees to fourteen places with the actual value

$$
\log 2=0.693147180559945 \text {. }
$$

Grateful acknowledgement is extended to Mr. V. Galin and Mr. A. Grossman, and especially to Miss M. Robinson for her assistance in the preparation of the manuscript.

New York City 